\title{
Green Energy Efficient Routing with Deep Learning Based Anomaly Detection for Internet of Things (IoT) Communications
}

\author{
E. Laxmi Lydia ${ }^{1}$, A. Arokiaraj Jovith ${ }^{2}$, A. Francis Saviour Devaraj ${ }^{3}$, Changho Seo ${ }^{4, *}$ and \\ Gyanendra Prasad Joshi ${ }^{5, *}$ (D)
}

1 Department of Computer Science and Engineering, Vignan's Institute of Information Technology (Autonomous), Visakhapatnam 530049, Andhra Pradesh, India; elaxmi2002@yahoo.com

2 Department of Information Technology, College of Engineering and Technology, SRM Institute of Science and Technology, Kattankulathur 603203, Tamil Nadu, India; arokiara@srmist.edu.in

3 Department of Computer Science and Engineering, Kalasalingam Academy of Research and Education, Krishnankoil 626128, Tamil Nadu, India; francis@klu.ac.in

4 Department of Convergence Science, Kongju National University, Gongju 32588, Korea

5 Department of Computer Science and Engineering, Sejong University, Seoul 05006, Korea

* Correspondence: chseo@kongju.ac.kr (C.S.); joshi@sejong.ac.kr (G.P.J.); Tel.: +82-2-6935-2481 (G.P.J.)

check for

updates

Citation: Lydia, E.L.; Jovith, A.A.; Devaraj, A.F.S.; Seo, C.; Joshi, G.P. Green Energy Efficient Routing with Deep Learning Based Anomaly

Detection for Internet of Things (IoT) Communications. Mathematics 2021, 9 500. https://doi.org/10.3390/ math9050500

Academic Editor: Vladimir M. Vishnevsky

Received: 28 December 2020

Accepted: 21 February 2021

Published: 1 March 2021

Publisher's Note: MDPI stays neutral with regard to jurisdictional claims in published maps and institutional affiliations.

Copyright: (c) 2021 by the authors. Licensee MDPI, Basel, Switzerland. This article is an open access article distributed under the terms and conditions of the Creative Commons Attribution (CC BY) license (https:/ / creativecommons.org/licenses/by/ $4.0 /)$.

\begin{abstract}
Presently, a green Internet of Things (IoT) based energy aware network plays a significant part in the sensing technology. The development of IoT has a major impact on several application areas such as healthcare, smart city, transportation, etc. The exponential rise in the sensor nodes might result in enhanced energy dissipation. So, the minimization of environmental impact in green media networks is a challenging issue for both researchers and business people. Energy efficiency and security remain crucial in the design of IoT applications. This paper presents a new green energyefficient routing with DL based anomaly detection (GEER-DLAD) technique for IoT applications. The presented model enables IoT devices to utilize energy effectively in such a way as to increase the network span. The GEER-DLAD technique performs error lossy compression (ELC) technique to lessen the quantity of data communication over the network. In addition, the moth flame swarm optimization (MSO) algorithm is applied for the optimal selection of routes in the network. Besides, DLAD process takes place via the recurrent neural network-long short term memory (RNN-LSTM) model to detect anomalies in the IoT communication networks. A detailed experimental validation process is carried out and the results ensured the betterment of the GEER-DLAD model in terms of energy efficiency and detection performance.
\end{abstract}

Keywords: Internet of Things; deep learning; anomaly detection; energy efficiency; routing

\section{Introduction}

The modern world adopts recent communication technologies to make a complete network coverage system and extended the intelligent object count which is correlated to the deployed system. One of the effective patterns named Internet of Things (IoT) is capable of increasing the volume of data generated from the Internet by connecting users and modern tools. Moreover, the IoT faces some issues, especially in location and time while connecting to humans and machines [1]. Moreover, IoT intends to achieve Quality of Service (QoS) such as energy, delay, throughput, etc. Bandwidth is one of the significant sources in the IoT platform, and appropriate management is essential to accomplish maximum QoS. Furthermore, the requirement for multimedia facilities in IoT platform is enhanced intensely. It finds useful in smart grids, smart homes, etc. [2,3]. Various services in IoT have distinct characteristics and QoS mechanisms. The fundamental strategy of IoT is to correlate one another with the help of the internet. In future, numerous applications might be developed and one among them is modern city. The exploitation of IoT has been increased rapidly and some of them are food, textiles, organization, transportation, academics, entertainment, and so forth. 
Due to the applicability of various communication models, IoT is applied in healthcare, army services, organizational fields, mobile communications, etc. The quantity of data balanced by IoT has not been presented due to insufficient storage space, request rate, and concentration of a tool. As persistent communication is provided, data management and its flow get varied by means of time. Numerous data and allocation services are emanated for IoT users by simple requests and responses via end-to-end servers as well as gateways.

In IoT model, low power and lossy network (LLN) comprised of numerous nodes with robust limitation of storage space, computation ability capacity, and energy. Based on this approach, nodes from IoT systems have diverse hardware abilities and application domains. The application of IoT is depicted in Figure 1, where the nodes are connected to the Internet. Unlike, alternate nodes from similar systems, because of the hardware constraints, a direct Internet connection is not possible and necessities the application of Internet-connected nodes to apply the physical services.

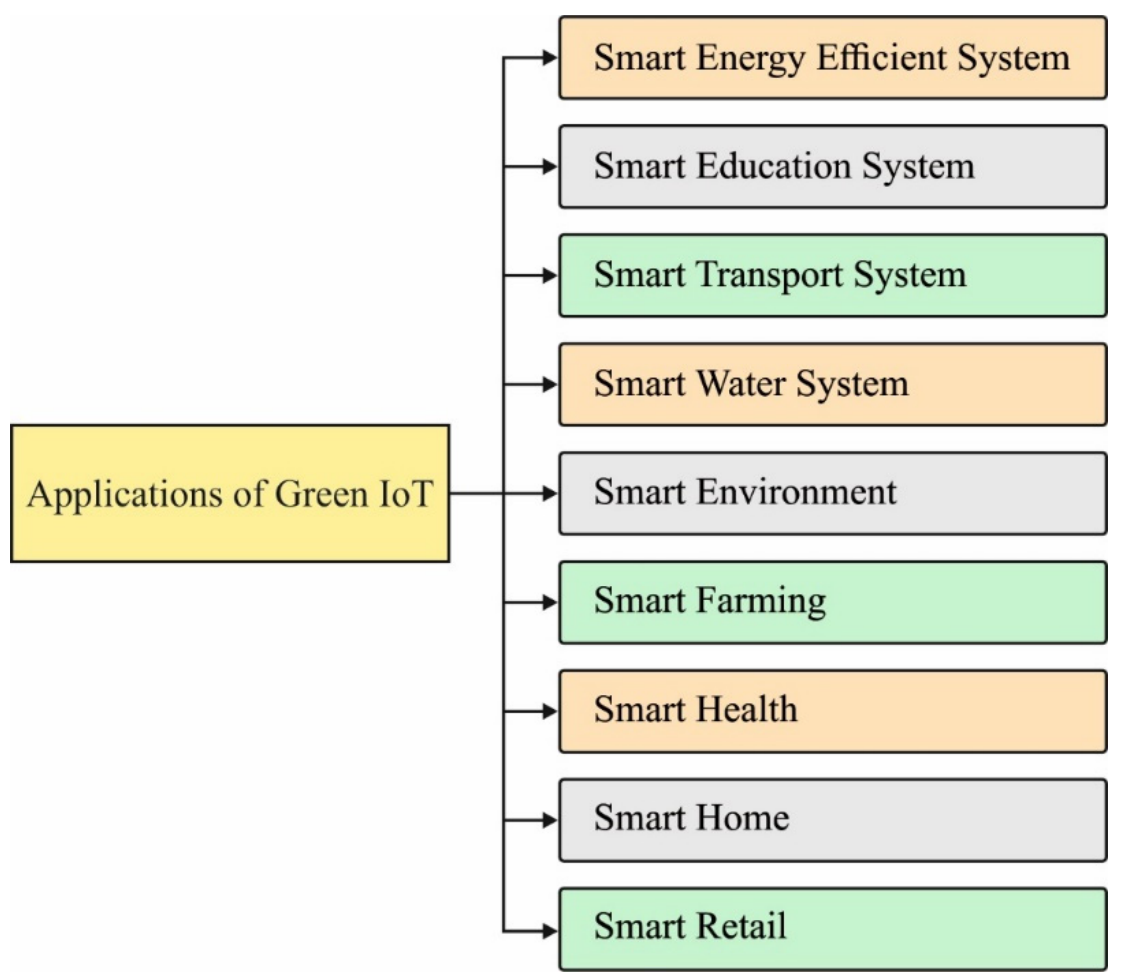

Figure 1. The Application of IoT.

Form the content of low-power IoT systems, the routing protocols are classified as 2 types on the basis of route developing strategies namely, Proactive and Reactive. Initially, proactive protocols simulate the deployment of routes between nodes with no advanced data message transmission. In general, route creation is accelerated by gateway nodes by gathering data from alternate system (Multipoint-to-Point (MP2P) traffic). For this purpose, this kind of protocol has been applied extensively by periodical data collecting models. The significant module of proactive routing is IPv6 Routing Protocol (RPL). Unlike, reactive protocols make routes if a node decides to forward a message to the receiver. Therefore, this kind of protocol is induced for a non-periodical traffic domain, in which a node transfers a message to some other destination (Point-to-Point (P2P) traffic).

The recently established and related reactive protocol for LLN is referred to be LOADng [4]. In the meantime, the increased IoT objectives have reasonable cybersecurity problems due to the presence of attacks. In recent times, the deployment of Deep Learning (DL) and hardware system provides a way to predict the traffic state, data offloading, and cyber-attacks in the edge system. Adopting DL methods are highly essential and significant in several applications where MEC offers the decisive advantage for traffic 
prediction and intrusion detection as huge data has been produced by IoT devices activates deep models to understand the workflow when compared with shallow methods. Feature learning with deep neural networks have shown considerable developed in the recent times $[5,6]$.

Though several coding schemes as well as routing techniques are available in the literature, they are not integrated to one another to develop an effective framework for green IoT. Besides, the design of new anomaly detection techniques is yet to be deeply explored. This paper develops a novel Green Energy Efficient Routing with Deep Learning based Anomaly Detection (GEER-DLAD) technique for IoT applications. The presented model permits the IoT devices to exploit energy efficiency in such a way to rise the network span. The GEER-DLAD technique accomplishes the error lossy compression (ELC) model to mitigate the quantity of data over the network. Moreover, moth flame swarm optimization (MSO) algorithm based routing process takes place in the network. In addition, DLAD process takes place via the recurrent neural network-long short term memory (RNN-LSTM) model. The integration of the GEER with anomaly detection, compression process, and energy efficient routing process shows the novelty of the work. In order to determine the supremacy of the GEER-DLAD model, a series of simulations were carried out.

\section{Related Works}

This section performs a detailed review of energy efficient routing protocols and data reduction techniques available in the literature.

\subsection{Existing Routing}

In Yi et al. [7], developers have related LOADng with RPL in applications with diverse traffic patterns. Based on the attained results, it is clear that LOADng outperformed RPL in point-to-multipoint (P2MP) as well as P2P traffic scenes. However, in MP2P, RPL has projected considerable outcomes. Moreover, the proactive properties of RPL create a better environment for data gathering applications. Unlike, LOADng is capable to perform better with comprehensive traffic; however, it has projected a maximum delay due to the requirement of examining route discovery. The working function of LOADng in IoT domains with P2P as well as MP2P traffic pattern as defined in Sobral et al. [8]. The influence of route selection in LOADng has been studied extensively in Sobral et al. [9]. An enhancement of LOADng is presented in Hossain et al. [10]. Therefore, Neighbor-Disjoint Multipath for LOADng (LOADng + NDM) shows a new multipath mechanism applied for LOADng and decides to enhance network consistency and QoS. A small path has been initialized from the transmitter and receiver node. Followed by, it also manages to develop collective backup routes by eliminating the nodes.

Araújo et al. [11] projected the enhancement routine measure for RPL in IoT platforms with heterogeneous tools. Fuzzy systems apply the routing measures in the network process using the application demands. Hence, the performance results have shown that the newly developed models are capable to resolve the basic RPL measures by means of power utilization as well as Packet Delivery Ratio (PDR). The routing protocol for IoT systems depends upon the routing measures as depicted in Machado et al. [12]. Moreover, Routing by Energy and Link quality (REL) projects the application of Ad-hoc On-demand Distance Vector (AODV) that decides to enhance the scalability and energy efficiency. Then, a protocol applies weakLinks approach to find the connections with limited quality over a path where the route development with maximum weakLinks is eliminated. Afterward, REL surpassed the AODV with respect to delay, PDR, and network survival rate.

Araújo et al. [13] projected a solution based smartphone to enable the interoperability of IoT tools. Developers have presented a structure where a smartphone collects huge communications interfaces to be operated as a gateway for diverse techniques. Hence, the software-based model applies the default execution of various standards. A special testbed is developed to estimate the solution by means of storage space, CPU, and power consumption. In Javaid et al. [14], developers have introduced solutions for gateway 
identification in Mobile Ad Hoc Networks (MANETs). This approach depends upon the application of periodical control messages and assumes the networks with mobile nodes which is ineffective for low-power IoT devices. Hence, the working function of solutions is determined by means of PDR as well as end-to-end delay.

\subsection{Existing Data Compression Approaches}

Various data reduction approaches for energy consumption of IoT has been deployed in the state of art. In Tayeh et al. [15], researchers have introduced a data reduction approach according to the dual prediction. The merits of the prediction model are that the edge forecasts the sensed value with no requirement of radio communication is processed till reaching the predefined threshold value. In Alieksieiev [16], developers have presented a data aggregation model for input data stream from IoT relied monitoring networks. The accomplished results have implied that it is applicable to gain a compression to the greater iterations on temperature data. In Azar et al. [17], researchers have projected data compression mechanism which is advantageous of temporal correlation in gathered data. It depends upon elegant and effective processing in 1D Discrete Wavelet Transform (DWT) by lifting models as well as Differential Pulse Code Modulation (DPCM).

Compressive Sensing (CS) as well as transform domain compression mechanism are prominently applied for images and multivariate time series compression in IoT domains. In Gaeta et al. [18], developers have employed multi-signal compression mechanism on the basis of fuzzy transform. It is utilized on multi-signal surrounding data gathered using Wireless Sensor Network (WSN) and limited the data to certain extent. In Cheng et al. [19], developers have presented 2-D lifting wavelet transformation for the purpose of compressing multi-signal data gathered from various sensor nodes. Hence, the newly developed model applies Haar wavelet and accomplished a better compression ratio as well as recovery accuracy. Transform domain compression is classified by the potential of recovering data. Besides, CS definition is generated as effective model energy-proficiency in IoT modalities. In addition, some context-adaptive binary arithmetic coding (CABAC) technique and their extended versions are available in $[20,21]$.

\section{The Proposed GEER-DLAD Model}

The overall system architecture of the presented GEER-DLAD model is depicted in Figure 2. As shown in figure, the IoT devices continuously gather the data. Then, they are compressed by the use of ELC technique. Subsequently, the IoT devices executed the routing technique using the MSO algorithm and select the optimal route to the destination. Upon the election of routes, the compressed data will be forwarded towards the time. At the time of data transmission, the DLAD technique is applied to detect the occurrence of anomalies in IoT communication networks.

\subsection{ELC Based Data Reduction Technique}

Initially, the IoT devices execute the data collection process to gather the data regarding the target environment. A lightweight model of compression named ELC is projected in [22]. It is a robust error-bounded lossy compression technique for High Performance Computing (HPC) domains. The actual SZ model compresses the incoming data files in binary form; it is composed of data structure and data types. In this literature, the SZ approach has been adopted for IoT tools by assuming the floating data type and eliminate alternate types that reduce the code size and simple execution. Hence, the strategy behind selecting SZ for IoT domains are listed below:

- $\quad \mathrm{SZ}$ enables compression of multivariate time series with distinct characteristics and varying scales.

- $\quad \mathrm{SZ}$ activates the data loss management with the help of error bound mechanism.

- $\quad \mathrm{SZ}$ results in maximum compression ratio when compared with multidimensional transform domains. 


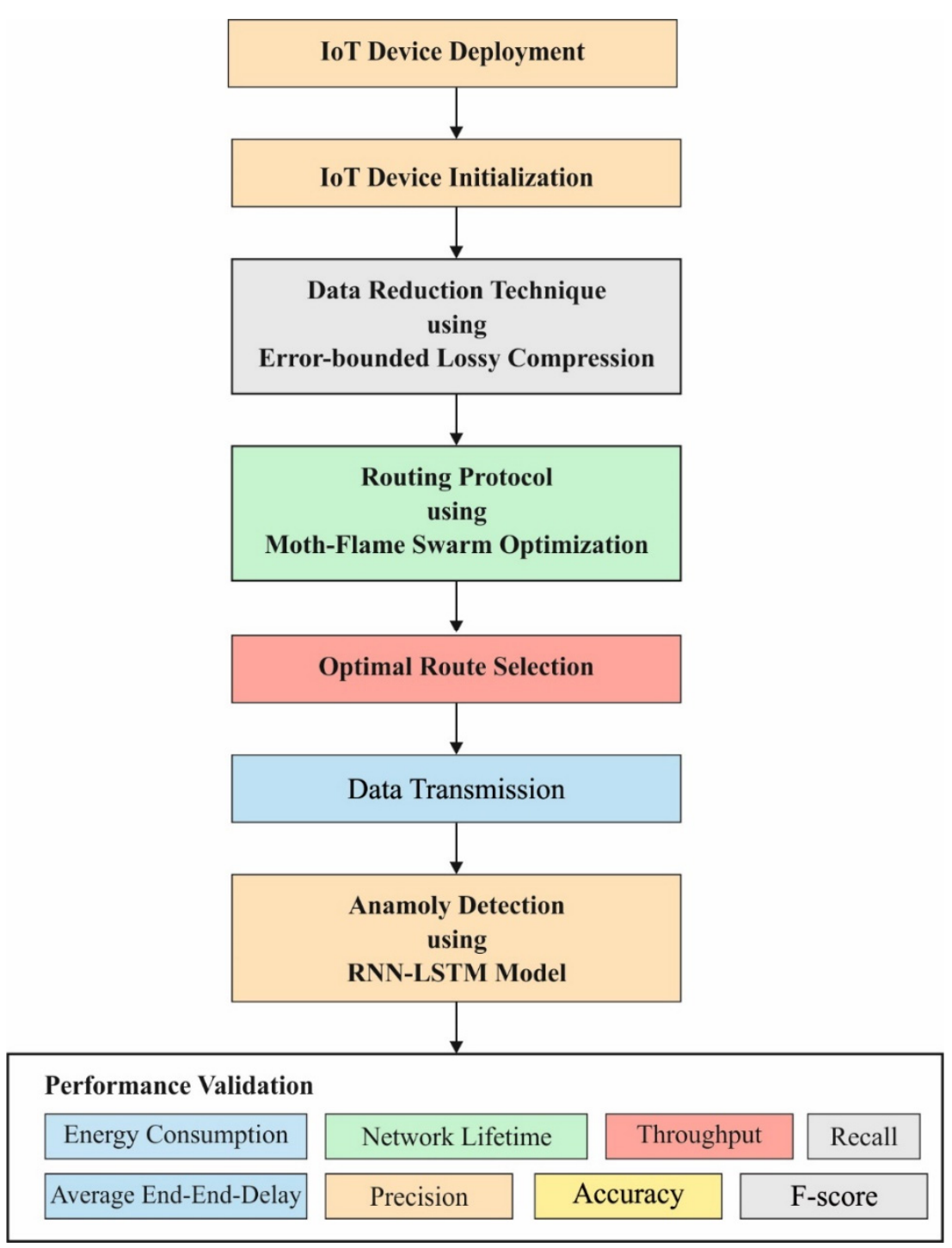

Figure 2. The overall working process of GEER-DLAD model.

The gathered data are $\mathrm{M} \times \mathrm{N}$ array, where $\mathrm{M}$ and $\mathrm{N}$ define the reading and feature count. For instance, assume a sensor movement gathered from gyroscope and accelerometer metrics for 3 coordinate axes over a period $\mathrm{P}$. At this point, $\mathrm{M}$ is allocated as 128 , and $\mathrm{N}$ is 6. Initially, 2-D array is changed into 1-D array. Next, a flattened array undergoes compression with the help of lossy SZ method. At last, final binary array has been sent to the edge.

The SZ compressor is started by reducing 1-D array under the application of adaptive curve-fitting methods [23]. The optimal step applies 3 prediction technologies namely, Preceding Neighbor Fitting (PNF), Linear-Curve Fitting (LCF), and Quadratic-Curve Fitting (QCF). The variations among the 3 schemes of precursor data points has to fit the actual value. Moreover, the nearest approximation has been provided as a result. It is apparent that the fitted data is converted as integer quantization and subjected to the encoding phase with the help of the Huffman tree. In case of error bound, absolute error bound is applied where compression and decompression errors are restricted within absolute error.

\subsection{MSO Based GEER Technique}

Next to the data reduction process, the compressed form of the collected data needs to be routed to the destination. To determine the optimal choices of routes, MSO based GEER technique is applied. The MSO algorithm is inspired by the process of choosing the 
optimal discriminating features of mitosis cells. In general, Moth is a bug that belongs to the butterfly family called Lepidoptera. Around 160,000 moth species exist globally, and it is visible at night-time. By comparing moth and alternate species, phototaxis and Levy flight is considered to be important.

\subsubsection{Phototaxis}

The basic strategy of the moth is that; it often moves around the light named phototaxis. There is no accurate method for phototaxis and is composed of the main hypotheses to define the phototaxis model. Followed by, it is meant to be a significant hypothesis in the celestial direction that has been executed in transverse orientation at the flying time. At the same time, the angle is initialized from the light source and the moth is directed, however, it is not applicable to acquire the transform, as the celestial object is found to be external distance. Thus, it travels to the light source, since moths create flight orientation to gain the best location [24]. A spiral-path has been developed to move closer to the light source.

\subsubsection{Levy Flight}

Heavy-tailed, non-Gaussian statistics are examined as common methods under diverse applications. Alternatively, Drosophila indicates a Levy flight, however, the flight is approximated as energy supply with feature exponent with 3/2. Generally, the Levy distribution is expressed in Equation (1),

$$
L(s) \sim|s|^{-\beta}
$$

where $1<\beta \leq 3$ denotes an index.

Basically, moths have the properties closer to optimal one and fly in a Levy flight mechanism approximately. Else, it instructs the position and uses Levy flight by means of Equation (2), however, moth is insisted using Equation (2):

$$
X_{j}^{a+1}=X_{j}^{a}+\delta L(s)
$$

where, $X_{j}^{a}$ and $X_{j}^{a+1}$ refers to the original and refreshed location at generation $a$, however the current generation is implied as $a$. It is accomplished from Levy flight as represented by $L(s)$. In problems of interest, scale factor defines the variable $\delta$. Hence, equation to $\delta$ is illustrated in Equation (3):

$$
\delta=W_{\max } / a^{2}
$$

Then, $W_{\max }$ denotes maximum walk step and value for $W_{\max }$ depends upon the available problem. $L(s)$ from above equation is reformed as,

$$
L(s)=\frac{(\alpha-1) \Gamma(\alpha-1) \operatorname{sm}\left(\frac{\pi(\alpha-1)}{2}\right)}{\pi s^{\alpha}}
$$

In $s$ is meant to be higher than $0 . \Gamma(x)$, indicates a gamma function. In prior to allocating from $L(s)$ with $\alpha=1.5$ moths Levy flight has been initialized.

\subsubsection{Fly Straight}

The present moths are isolated from light source that flutters in straight line in the direction of light. This function of moth $j$ is devised as,

$$
X_{j}^{a+1}=\lambda \times\left(X_{j}^{a}+\varphi\left(X_{b e s t}^{a}-X_{j}^{a}\right)\right)
$$

where $X_{\text {best }}^{a}$ defines the optimal moth at $a$ generation, $\lambda$ indicates a scale factor. $\varphi$ denotes acceleration factor. 
Else, moth will move away from light source to final position. Hence, the final location of moth $j$ is offered in Equation (6),

$$
X_{j}^{a+1}=\lambda \times\left(X_{j}^{a}+\frac{1}{\varphi}\left(X_{\text {best }}^{a}-X_{j}^{a}\right)\right)
$$

For integrity, the location of moth $\mathrm{j}$ is insisted by incomplete percentage. The original, refreshed, and best location of moth is projected as $X_{j}, X_{j, \text { new }}$ and, $X_{\text {best }} . \lambda$, tried the techniques with enhanced population diversity. Figure 3 shows the flowchart of MSO techniques.

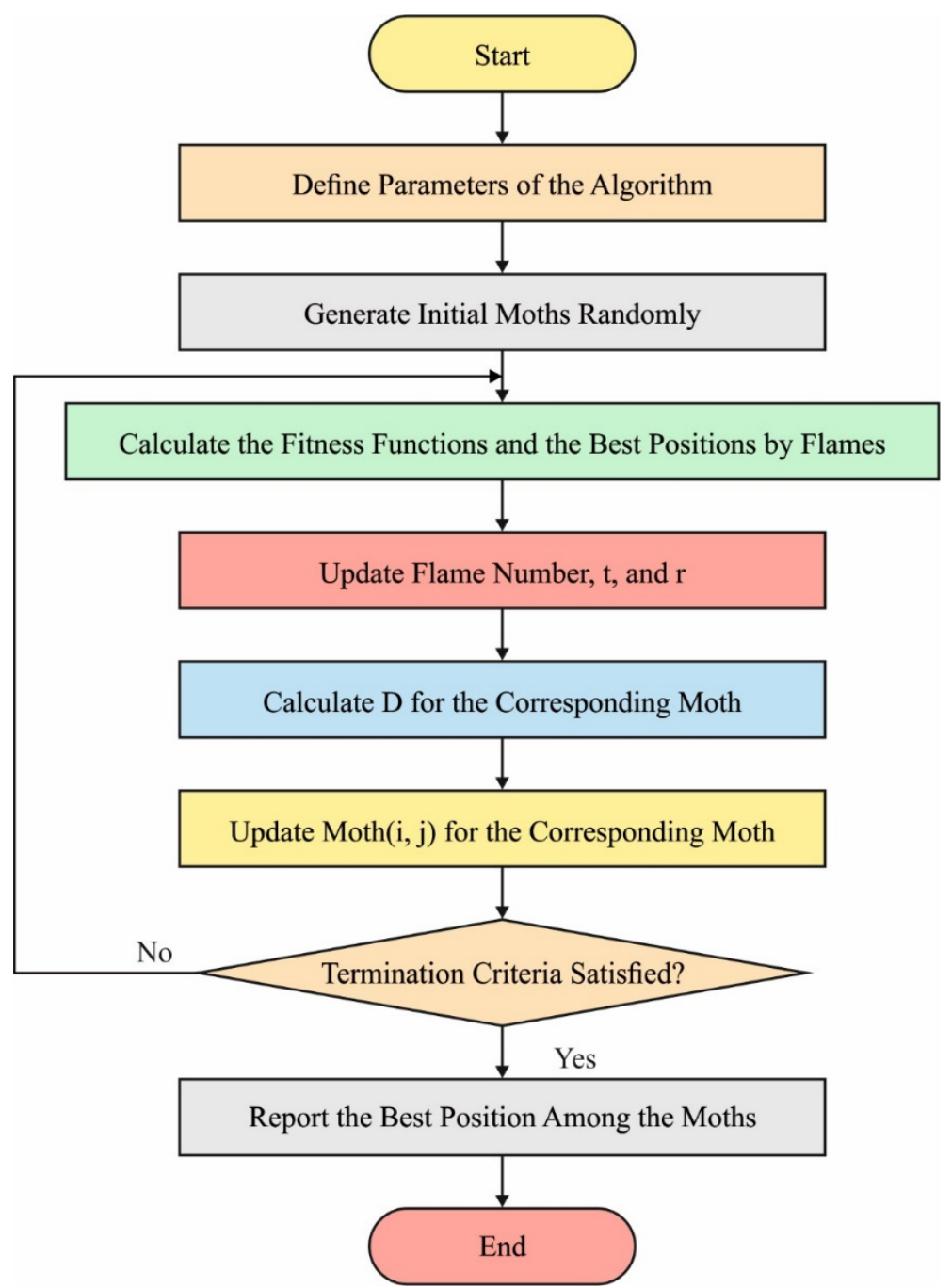

Figure 3. The Flowchart of MSO algorithm.

Then, FF values of the GEER technique using MSO is projected for generating a competent routing path between two IoT devices are given below. Afterward, the distance (P) traversed with gateways are defined in Equation (7).

$$
\mathrm{P}=\sum_{\mathrm{p}=1}^{\mathrm{m}} \mathrm{dst}\left(\mathrm{l}_{\mathrm{q}}, \mathrm{NxtL}\left(\mathrm{l}_{\mathrm{q}}\right)\right)
$$


The values of gateway are the system is defined in Equation (8).

$$
\mathrm{H}=\sum_{\mathrm{Q}=1}^{\mathrm{m}} \operatorname{NxtLCount}\left(\mathrm{l}_{\mathrm{q}}\right)
$$

Routing is ensured by small traversal and minimum number of hops. Lastly, minimum crossing distance and hop results in maximum fitness value. Thus, solution with higher fitness value is assumed to be optimal solution and the proposed FF is depicted in Equation (9).

$$
\text { Routing_Fitness }=\frac{\mathrm{Z}_{1}}{\left(\mathrm{u}_{1} * \mathrm{P}+\mathrm{u}_{2} * \mathrm{H}\right)}
$$

where $\left(\mathrm{u}_{1}, \mathrm{u}_{2}\right) \in[0,1]$ such that, $\mathrm{u}_{1}+\mathrm{u}_{2}=1$ and $\mathrm{Z}_{1}$ indicates proportionality stable. Routing fitness utility is used for managing the complete distance and count hop in a system.

\subsection{RNN-LSTM Based DLAD Technique}

During the data transmission process, the DLAD task is performed using RNN-LSTM model to determine the existence of anomalies in the IoT networks. The LSTM model is mainly used for predicting the anomalies in RNN. It seems like a remarkable RNN with a hidden layer, in which a normal node of hidden layer is replaced with the help of memory cell as depicted in Figure 4. To compute the references of memory cell, subscript c is employed.

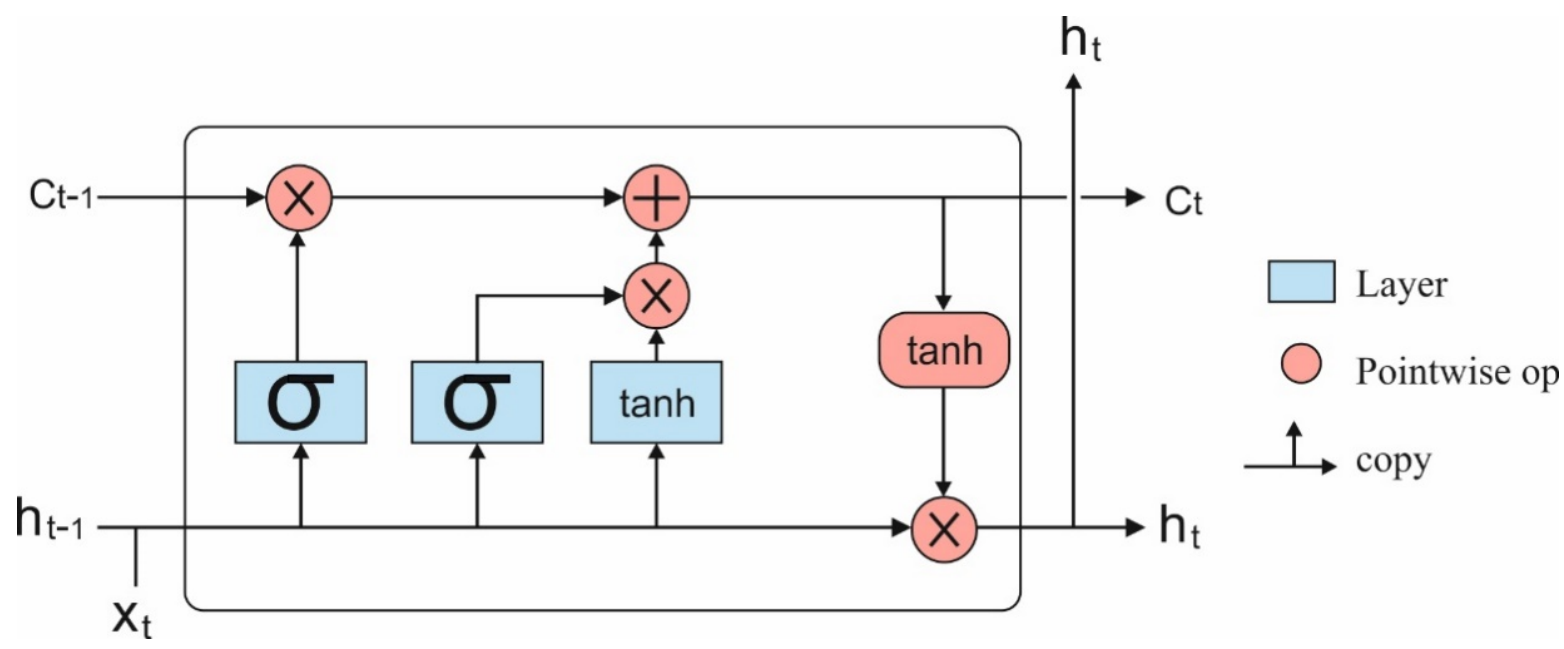

Figure 4. The Structure of LSTM.

The elegant recurrent $\mathrm{NN}$ is composed of long-term memory by means of weights. It is changed in training duration, and encode the general knowledge about a data. Moreover, short-term memory interms of ephemeral functions, which are passed from single node to next nodes. In this module, LSTM approach signifies an intermediate type of memory cell. The units of LSTM cells are enumerated and described as shown in the following. For example, s refers a vector with measure of $s_{\mathcal{C}}$ at each memory cell $c$ from a layer [25]. When subscript $\mathrm{c}$ is applied, then it assists in a single memory cell.

Input node: The labeled $g_{c}$, is considered as a node that gains activation from input layer $l^{(z)}$ in recent duration and from hidden layer at previous time step $h^{(z-1)}$.

- Input gate: The gates are considered as exclusive property of LSTM method. The gate mimics a sigmoidal input node, which acquires the activation from recent data point $l^{(z)}$ and from hidden layers previously. It is named as gate because of the multiplied value of some other node. When 0 is allocated as a value, then it is called gate while 
the flow of other node is cut off. And, when 1 is allocated, then all flows are conveyed. In addition, measure of input gate $u_{c}$ enhances the measure of input node.

- Internal state: The kernel points of memory cells are node $s_{\mathcal{C}}$ with linear activation called inner state of a cell. Hence, the interior state $s_{c}$ has self-connected recurrent edge in conjunction with fixed unit weight. Because of the edge of time steps with same weight, error flows across time steps under the inexistence of exploding. It is called a constant error container. In case of vector function, upgrade the internal state as $s^{(z)}=g^{(z)} \odot u^{(z)}+s^{(z-1)}$ where $\odot$ denotes point-wise multiplication task.

- $\quad$ Forget gate: The gates $f_{c}$ has been developed. It is capable of learning the content of interior state. It is highly suitable in prominent networks. Under the application of forget gates, forward pass in internal state can be measured as given below:

$$
s^{(z)}=g^{(z)} \odot u^{(z)}+f^{(z)} \odot s^{(z-1)}
$$

- $\quad$ Output gate: Value $v_{c}$ is emanated by memory cell that has been assumed as value of internal state $s_{c}$ and enhanced by final gate $o_{c}$. Moreover, it is considered that internal state is executed using tanh activation function, as the final outcome of a cell with same dynamic range is produced. However, in NN, solved linear units are composed of maximum dynamic range which helps in simple training. Therefore, it is reasonable for nonlinear function in which internal state is eliminated.

Basically, the input node is called as $g$. Here, gates are known $y_{\text {in }}$ and $y_{\text {out }}$ but the conflicts of $y$ show a better result in the ML approach. Based on the comprehensibility, the convention is classified and applies $u, f$, and $o$ defines input, forget, and outcome gates.

The peephole relations passed from internal state to input and outcome gates of the same node lacks primary rules to be applied in outcome gate. Consider a network to estimate objects and emits required results when $n$ objects are viewed. Moreover, the system identifies different numbers of activation to the internal state. Such activations have emerged within the internal state $s_{c}$ with constant error container and enhanced. When $n$th object is considered, the network requires to find the internal state. This is accomplished by using outcome gates $o_{\mathcal{C}}$ to learn the satisfying form of internal state $s_{\mathcal{C}}$. Thus, $s_{\mathcal{C}}$ should be an input to $o_{c}$. The estimation of LSTM depends upon memory cells appropriately. The consecutive process is implemented for all iterations. The expressions applied for recent LSTM with forget gates are depicted as:

$$
\begin{gathered}
g^{(z)}=\varphi\left(W^{g l} l^{(z)}+W^{g h} h^{(z-1)}+b_{g}\right) \\
u^{(z)}=\sigma\left(W^{u l} l^{(t)}+W^{u h} h^{(z-1)}+b_{u}\right) \\
f^{(z)}=\sigma\left(W^{f l} l^{(t)}+W^{f h} h^{(z-1)}+b_{f}\right) \\
o^{(z)}=\sigma\left(W^{o l} l^{(t)}+W^{o h} h^{(z-1)}+b_{o}\right) \\
s^{(z)}=g^{(z)} \odot u^{(u)}+s^{(z-1)} \odot f^{(t)} \\
h^{(z)}=\phi\left(s^{(z)}\right) \odot o^{(t)} .
\end{gathered}
$$

The measure of hidden layer of LSTM at time $z$ is depicted as vector $h^{(z)}$, as $h^{(z-1)}$ defines the measure of memory cell in hidden layer at previous time. Assume the function with forget gate, even though the peephole connections are absent. The processing step becomes simple for LSTM with no forget gates and accomplished with $f^{(z)}=1$ to every $z$. However, LSTM is the activation function to $g$ and sigmoid $\sigma$.

Simultaneously, by means of a forward pass, LSTM is known only when activation is induced for internal state. It is given that input gate has accomplished value 0 , and no activation function is accomplished. Followed by, LSTM has implemented maximum ability to understand long-range dependencies as relevant to simple RNNs. 


\section{Performance Validation}

The presented technique is implemented in a PC i5-8600k processor, GeForce 1050Ti, 4GB RAM, 16GB OS Storage and 250GB SSD File Storage. This section discusses the analysis of the result of the presented GEER-DLAD model interms of energy efficiency, lifetime, throughput, average delay, and detection performance. For validating the energyefficient performance, a series of comparative results analysis takes place with grasshopper optimization algorithm (GOA), glowworm swarm optimization algorithm (GSOA), multi-objective particle swarm optimization algorithm (MOPSOA), and particle swarm optimization algorithm (PSOA).

Table 1 and Figure 5 analyses the energy consumption results of the GEER-DLAD with existing methods with respect to distinct IoT devices. The resultant figure reported that the PSOA technique has resulted in inferior performance by exhibited higher energy consumption. Followed by, the MOPSOA has revealed slightly reduced energy consumption over PSOA. At the same time, the GSOA and GOA models have demonstrated closer energy consumption. However the presented GEER-DLAD model has accomplished better performance by obtaining reduced energy consumption over all the other compared methods. For instance, on the applied 10 IoT devices, the GEER-DLAD model has exploited a minimum energy consumption of $35 \mathrm{~mJ}$ whereas the GSOA, GOA, MOPSOA, and PSOA models have depicted higher energy consumption of $40 \mathrm{~mJ}, 43 \mathrm{~mJ}, 46 \mathrm{~mJ}$, and $55 \mathrm{~mJ}$ respectively. Simultaneously, on the given 50 IoT devices, the GEER-DLAD model has applied a minimum energy consumption of $105 \mathrm{~mJ}$ whereas the GSOA, GOA, MOPSOA, and PSOA methods have depicted higher energy consumption of $126 \mathrm{~mJ}, 135 \mathrm{~mJ}, 146 \mathrm{~mJ}$, and $165 \mathrm{~mJ}$ respectively. Concurrently, on the applied 100 IoT devices, the GEER-DLAD model has employed low power consumption of $160 \mathrm{~mJ}$ whereas the GSOA, GOA, MOPSOA, and PSOA models have illustrated maximum energy consumption of $174 \mathrm{~mJ}, 195 \mathrm{~mJ}, 213 \mathrm{~mJ}$, and $222 \mathrm{~mJ}$ respectively.

Table 1. The Result Analysis of Existing with Proposed GEER-DLAD Method in terms of Energy Consumption (mJ).

\begin{tabular}{cccccc}
\hline \multirow{2}{*}{$\begin{array}{c}\text { No. of IoT } \\
\text { Devices }\end{array}$} & GEER-DLAD & GSOA & GOA & MOPSOA & PSOA \\
\cline { 2 - 6 } & 35 & 40 & 43 & 46 & 55 \\
\hline 10 & 56 & 64 & 68 & 71 & 80 \\
\hline 20 & 72 & 85 & 92 & 98 & 107 \\
\hline 30 & 81 & 99 & 107 & 111 & 140 \\
\hline 40 & 105 & 126 & 135 & 146 & 165 \\
\hline 60 & 119 & 137 & 147 & 163 & 174 \\
\hline 70 & 125 & 150 & 164 & 177 & 186 \\
\hline 80 & 134 & 161 & 177 & 192 & 197 \\
\hline 90 & 145 & 166 & 189 & 198 & 211 \\
\hline 100 & 160 & 174 & 195 & 213 & 222 \\
\hline
\end{tabular}

Table 2 and Figure 6 investigates the network lifetime analysis of the GEER-DLAD model under distinct IoT devices. From the figure, it is showcased that the PSOA and MOPSOA models have demonstrated least network lifetime over the other methods. At the same time, GOA and GSOA models have exhibited even better network lifetime, but not superior to GEER-DLAD model. However, the GEER-DLAD model has exhibited improved network lifetime when compared with other models. For sample, on the applied 10 IoT devices, the GEER-DLAD model has obtained a higher network lifetime of 5600 rounds whereas the GSOA, GOA, MOPSOA, and PSOA models have exhibited reduced network lifetime of 5500, 5200, 4800, and 4500 rounds. Eventually, on the applied 50 IoT tools, 
the GEER-DLAD approach has gained a higher network lifetime of 4900 rounds whereas the GSOA, GOA, MOPSOA, and PSOA models have shown limited network lifetime of 4700, 4500, 4000, and 3600 rounds. Along with that, on the applied 100 IoT devices, the GEER-DLAD scheme has achieved a maximum network lifetime of 3800 rounds while the GSOA, GOA, MOPSOA, and PSOA schemes have showcased limited network lifetime of $3300,3100,2900$, and 2800 rounds.

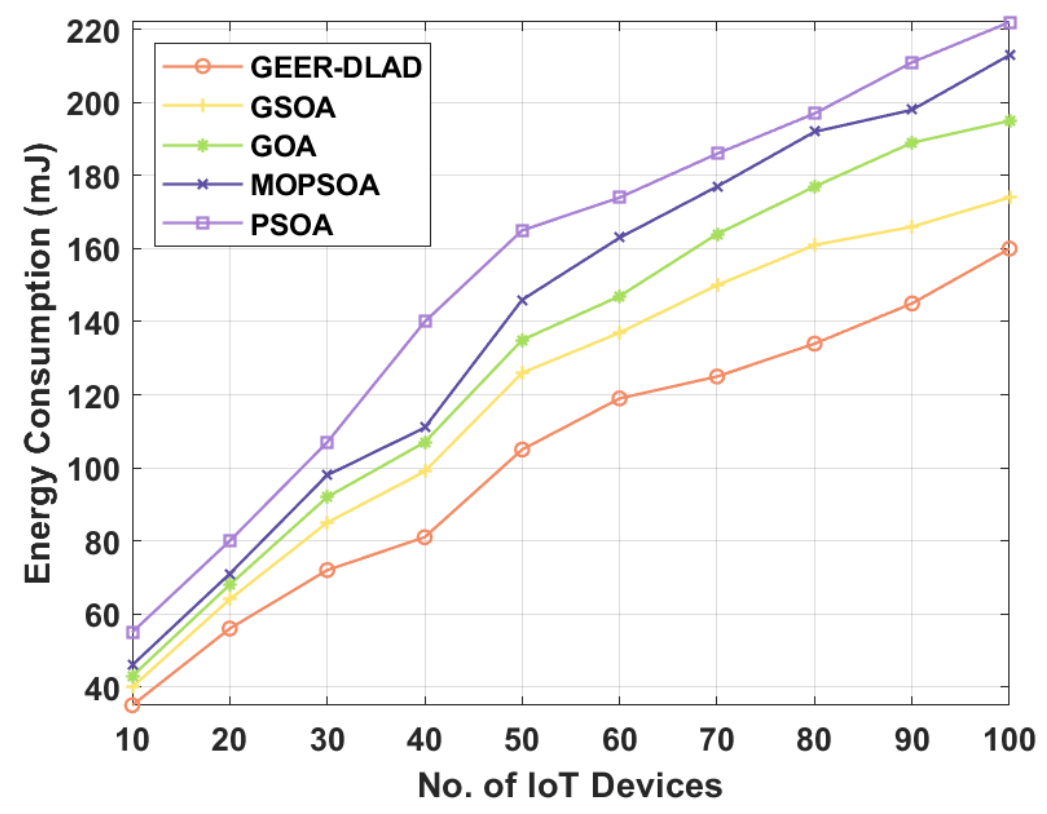

Figure 5. The Energy consumption analysis of GEER-DLAD model.

Table 2. The Result Analysis of Existing with Proposed GEER-DLAD Method in terms of Network Lifetime (Rounds).

\begin{tabular}{cccccc}
\hline \multirow{2}{*}{$\begin{array}{c}\text { No. of IoT } \\
\text { Devices }\end{array}$} & \multicolumn{5}{c}{ Network Lifetime (Rounds) } \\
\cline { 2 - 6 } & GEER-DLAD & GSOA & GOA & MOPSOA & PSOA \\
\hline 10 & 5600 & 5500 & 5200 & 4800 & 4500 \\
\hline 20 & 5500 & 5300 & 5000 & 4500 & 4300 \\
\hline 30 & 5300 & 5000 & 4800 & 4300 & 4200 \\
\hline 40 & 5100 & 4900 & 4700 & 4200 & 3800 \\
\hline 50 & 4900 & 4700 & 4500 & 4000 & 3600 \\
\hline 60 & 4600 & 4300 & 4200 & 3700 & 3500 \\
\hline 70 & 4400 & 4100 & 3900 & 3400 & 3300 \\
\hline 80 & 4100 & 3800 & 3500 & 3300 & 3200 \\
\hline 90 & 3900 & 3500 & 3300 & 3000 & 2900 \\
\hline 100 & 3800 & 3300 & 3100 & 2900 & 2800 \\
\hline
\end{tabular}

Table 3 and Figure 7 scrutinizes the throughput analysis of the GEER-DLAD model under various IoT devices. From the figure, it is portrayed that the PSOA and MOPSOA models have demonstrated least throughput over the other methods. Meantime, GOA and GSOA models have exhibited even better throughput, but not superior to GEER-DLAD model. However, the GEER-DLAD model has depicted improved throughput across the related methods. For instance, on the applied 10 IoT devices, the GEER-DLAD model has attained a higher throughput of $0.95 \mathrm{Mbps}$ while the GSOA, GOA, MOPSOA, and PSOA models have exhibited reduced throughput of $0.92 \mathrm{Mbps}, 0.91 \mathrm{Mbps}, 0.89 \mathrm{Mbps}$, 
and $0.85 \mathrm{Mbps}$. Followed by, on the applied 50 IoT devices, the GEER-DLAD model has obtained a maximum throughput of $0.84 \mathrm{Mbps}$ whereas the GSOA, GOA, MOPSOA, and PSOA models have illustrated minimal throughput of $0.67 \mathrm{Mbps}, 0.60 \mathrm{Mbps}, 0.55 \mathrm{Mbps}$, and $0.47 \mathrm{Mbps}$ iterations. Similarly, on the applied 100 IoT devices, the GEER-DLAD model has accomplished maximum throughput of $0.70 \mathrm{Mbps}$ whereas the GSOA, GOA, MOPSOA, and PSOA models have implied reduced throughput of $0.56 \mathrm{Mbps}, 0.48 \mathrm{Mbps}, 0.47 \mathrm{Mbps}$, and $0.38 \mathrm{Mbps}$.

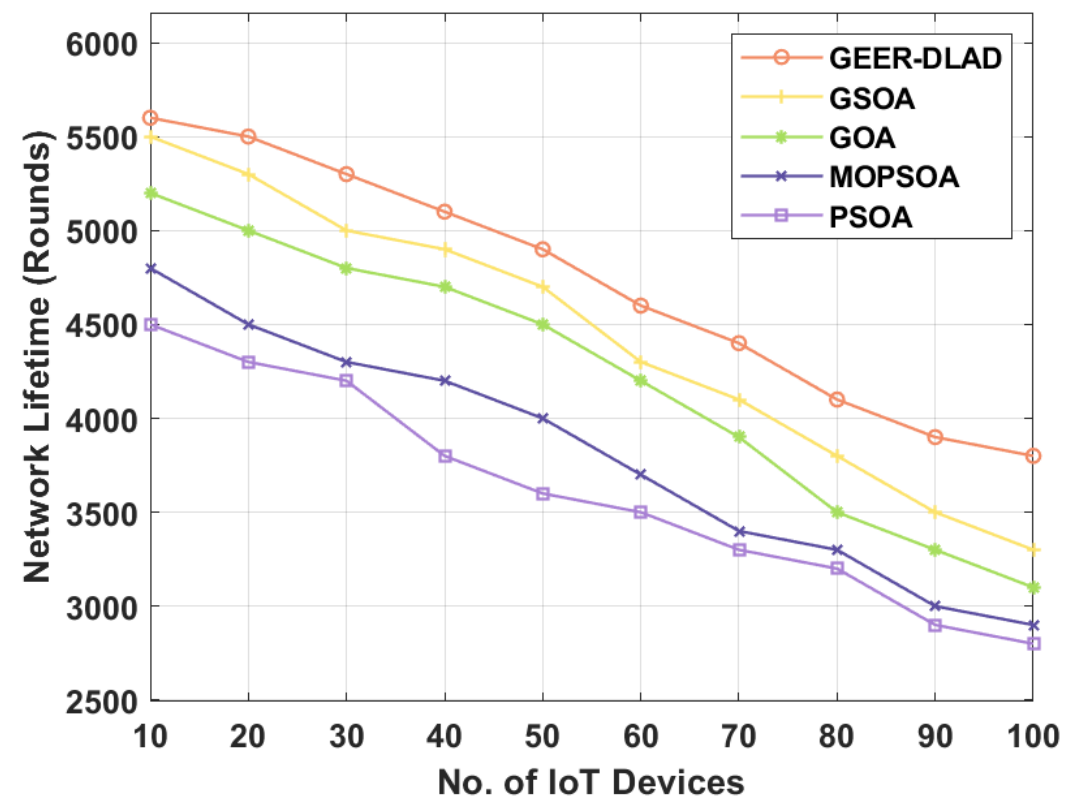

Figure 6. The Network lifetime analysis of GEER-DLAD model.

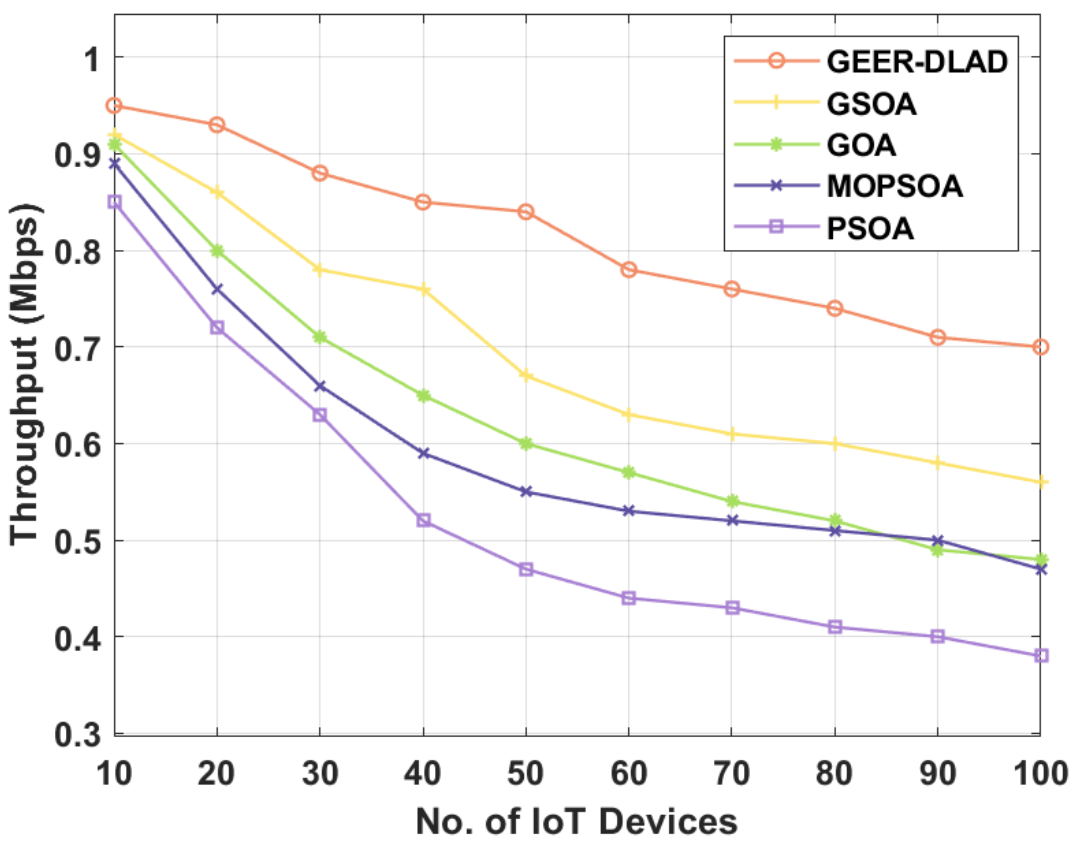

Figure 7. Throughput analysis of GEER-DLAD model. 
Table 3. The Result Analysis of Existing with Proposed GEER-DLAD Method in terms of Throughput (Mbps).

\begin{tabular}{cccccc}
\hline \multirow{2}{*}{$\begin{array}{c}\text { No. of IoT } \\
\text { Devices }\end{array}$} & GEER-DLAD & GSOA & GOA & MOPSOA & PSOA \\
\cline { 2 - 6 } & 0.95 & 0.92 & 0.91 & 0.89 & 0.85 \\
\hline 10 & 0.93 & 0.86 & 0.80 & 0.76 & 0.72 \\
\hline 20 & 0.88 & 0.78 & 0.71 & 0.66 & 0.63 \\
\hline 30 & 0.85 & 0.76 & 0.65 & 0.59 & 0.52 \\
\hline 40 & 0.84 & 0.67 & 0.60 & 0.55 & 0.47 \\
\hline 50 & 0.78 & 0.63 & 0.57 & 0.53 & 0.44 \\
\hline 70 & 0.76 & 0.61 & 0.54 & 0.52 & 0.43 \\
\hline 80 & 0.74 & 0.60 & 0.52 & 0.51 & 0.41 \\
\hline 90 & 0.71 & 0.58 & 0.49 & 0.50 & 0.40 \\
\hline 100 & 0.70 & 0.56 & 0.48 & 0.47 & 0.38 \\
\hline
\end{tabular}

Table 4 and Figure 8 examines the average delay results of the GEER-DLAD with existing methods with respect to distinct IoT devices. The resultant figure reported that the PSOA technique has resulted in poor performance by exhibited higher average delay. Followed by, the MOPSOA has revealed slightly reduced average delay over PSOA. At the same time, the GSOA and GOA models have depicted closer average delay. However the presented GEER-DLAD model has accomplished better performance by gaining reduced average delay over all the other compared methods. For example, on the applied 10 IoT devices, the GEER-DLAD model has exploited a minimum average delay of $2.02 \mathrm{~s}$ and the GSOA, GOA, MOPSOA, and PSOA models have depicted higher average delay of $2.22 \mathrm{~s}$, $2.32 \mathrm{~s}, 2.34 \mathrm{~s}$, and $2.97 \mathrm{~s}$ correspondingly. At the same time, on the applied 50 IoT devices, the GEER-DLAD model has exploited a minimum average delay of $5.64 \mathrm{~s}$ and the GSOA, GOA, MOPSOA, and PSOA models have depicted higher average delay of $5.86 \mathrm{~s}, 6.13 \mathrm{~s}$, $6.38 \mathrm{~s}$, and $9.87 \mathrm{~s}$ respectively. Meantime, on the applied 100 IoT devices, the GEER-DLAD model has employed a least average delay of $6.55 \mathrm{~s}$ whereas the GSOA, GOA, MOPSOA, and PSOA models have demonstrated optimal average delay of $8.75 \mathrm{~s}, 8.87 \mathrm{~s}, 9.24 \mathrm{~s}$, and 11.95 s respectively.

Table 4. Result Analysis of Existing with Proposed GEER-DLAD Method in terms of Average Delay (s).

\begin{tabular}{cccccc}
\hline \multirow{2}{*}{$\begin{array}{c}\text { No. of IoT } \\
\text { Devices }\end{array}$} & GEER-DLAD & GSOA & GOA & MOPSOA & PSOA \\
\cline { 2 - 6 } & 2.02 & 2.22 & 2.32 & 2.34 & 2.97 \\
\hline 10 & 2.14 & 2.75 & 3.21 & 3.83 & 5.33 \\
\hline 20 & 2.96 & 3.86 & 3.85 & 4.91 & 6.27 \\
\hline 30 & 3.73 & 4.12 & 4.91 & 5.77 & 7.97 \\
\hline 40 & 5.64 & 5.86 & 6.13 & 6.38 & 9.87 \\
\hline 50 & 5.86 & 6.85 & 6.87 & 6.92 & 9.23 \\
\hline 60 & 5.97 & 6.99 & 7.38 & 7.55 & 9.78 \\
\hline 70 & 6.64 & 7.65 & 7.81 & 8.47 & 10.85 \\
\hline 80 & 6.42 & 7.14 & 8.65 & 9.01 & 10.88 \\
\hline 90 & 6.55 & 8.75 & 8.87 & 9.24 & 11.95 \\
\hline 100 & & &
\end{tabular}




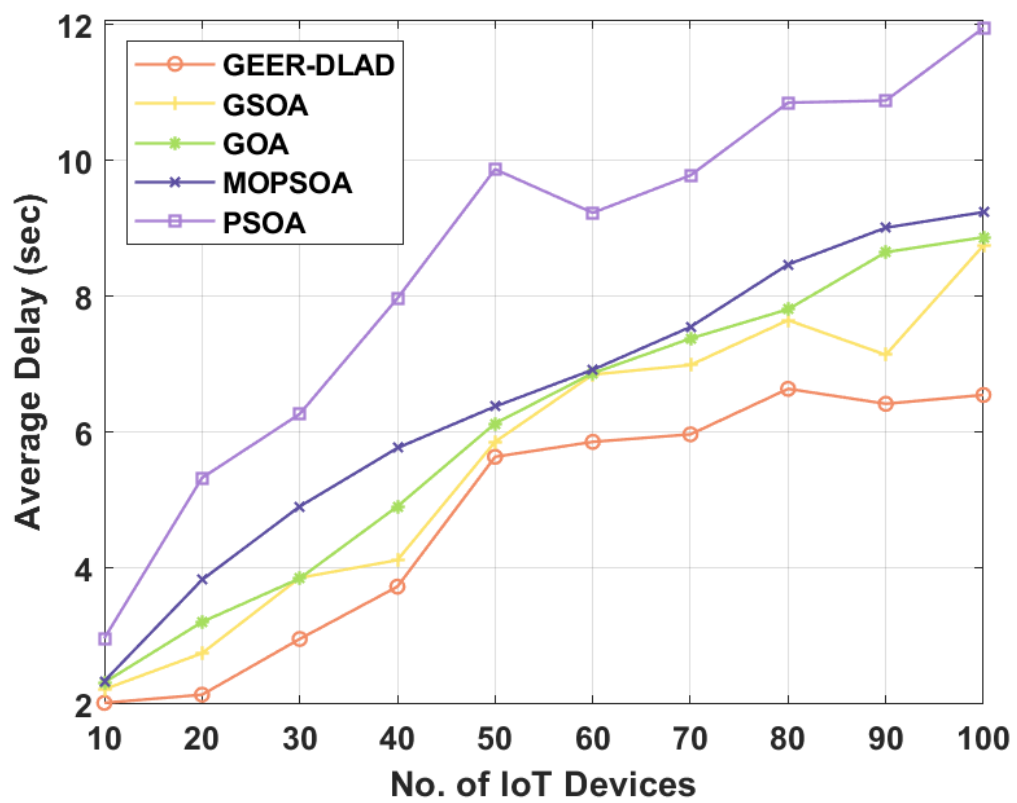

Figure 8. Average delay analysis of GEER-DLAD model.

In order to validate the anomaly detection performance of the RNN-LSTM model, a detailed simulation process was carried out on NSL-KDD dataset [26]. It includes a total of 45,927 samples under DoS attack, 995 samples under R2l attack, 11,656 samples under Probe attack, 52 samples under U2r attack, and 67,343 samples under Normal class.

Table 5 and Figure 9 illustrates the detection results of the RNN-LSTM model on the detection of distinct attack types. On the detection of DoS attack, the RNN-LSTM model has reported a precision, recall, F-measure, and accuracy of $96.15 \%, 97.17 \%, 96.36 \%$, and $96.58 \%$. Likewise, on the prediction of R21 attack, the RNN-LSTM method has shown a precision, recall, F-measure, and accuracy of $97.19 \%, 97.41 \%, 97.30 \%$, and $97.38 \%$. Besides, on the examination of Probe attack, the RNN-LSTM model has exhibited a precision, recall, F-measure, and accuracy of $98.08 \%, 98.02 \%, 97.91 \%$, and $97.89 \%$. Additionally, on the prediction of U2r attack, the RNN-LSTM model has implied a precision, recall, F-measure, and accuracy of $97.06 \%, 97.43 \%, 97.22 \%$, and $97 \%$. Furthermore, on the detection of normal attack, the RNN-LSTM model has showcased a precision, recall, F-measure, and accuracy of $96.71 \%, 97.20 \%, 97.09 \%$, and $97.13 \%$. Moreover, the average outcome of the RNN-LSTM model has attained maximum outcome of precision, recall, F-measure, and accuracy of $97.04 \%, 97.45 \%, 97.18 \%$, and $97.20 \%$.

Table 5. Result Analysis of Proposed RNN-LSTM Method.

\begin{tabular}{ccccc}
\hline Attack Type & Precision & Recall & F-Measure & Accuracy \\
\hline Dos & 96.15 & 97.17 & 96.36 & 96.58 \\
\hline R21 & 97.19 & 97.41 & 97.30 & 97.38 \\
\hline Probe & 98.08 & 98.02 & 97.91 & 97.89 \\
\hline U2r & 97.06 & 97.43 & 97.22 & 97.00 \\
\hline Normal & 96.71 & 97.20 & 97.09 & 97.13 \\
\hline Average & 97.04 & 97.45 & 97.18 & 97.20 \\
\hline
\end{tabular}




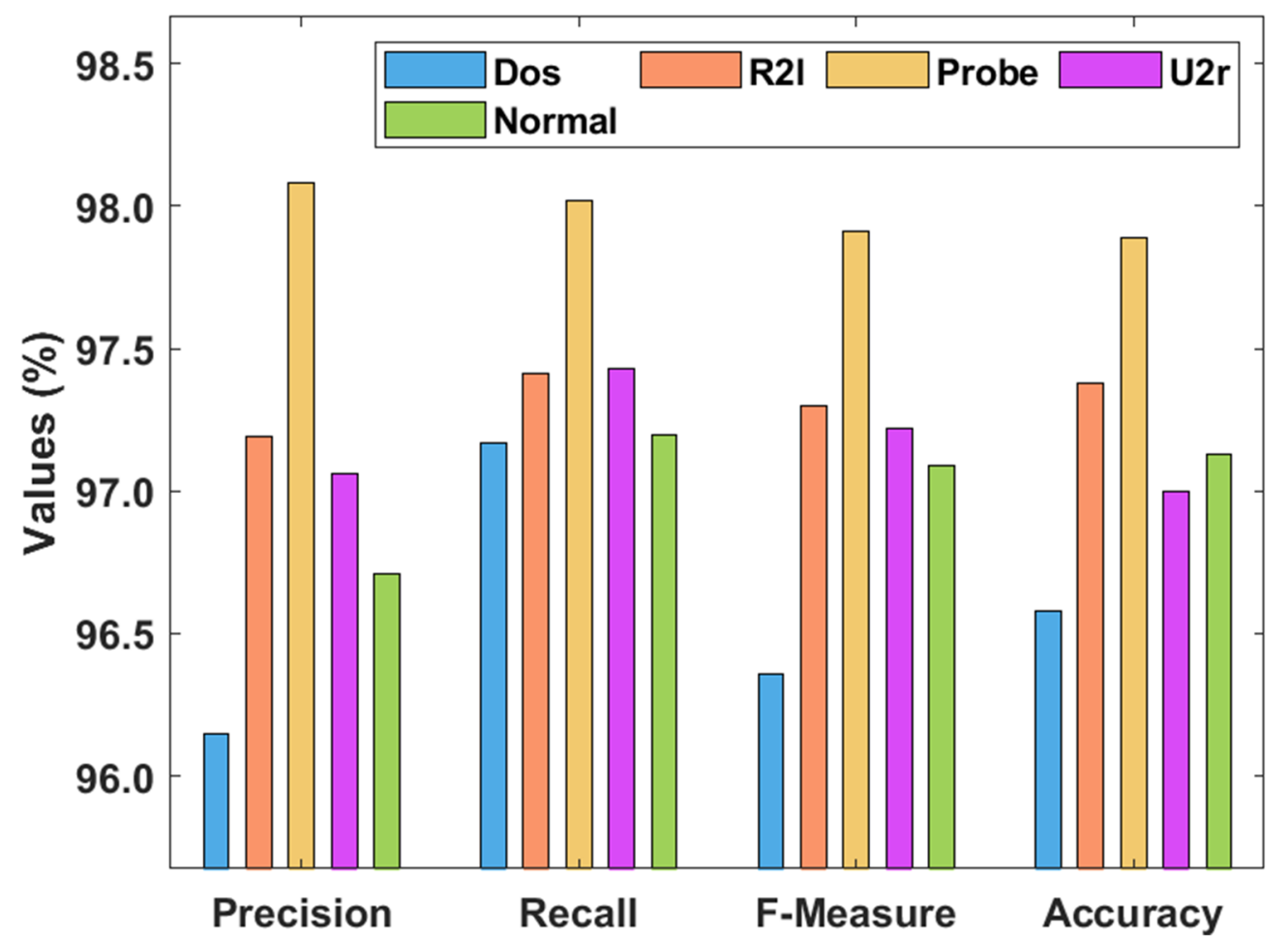

Figure 9. The Classifier results analysis of the RNN-LSTM model.

Table 6 and Figure 10 provides the comparative results of the RNN-LSTM model with previous techniques [27-29]. The experimental results implied that the AK-NN method has accomplished inferior results with the minimum precision of $92.19 \%$, recall of $93.76 \%$, F-measure of $92.92 \%$, and accuracy of $91.99 \%$. Similarly, the DL model has achieved a considerable precision of $93.54 \%$, recall of $94.87 \%$, F-measure of $94.12 \%$, and accuracy of $92.77 \%$. Afterward, the DPC-DBN model has achieved a reasonable precision, recall, Fmeasure, and accuracy of $95.12 \%, 94.99 \%, 95.08 \%$, and $94.98 \%$. Similarly, the DT model has attained competing for precision, recall, F-measure, and accuracy of $96.59 \%, 92.84 \%, 95.42 \%$, and $93.65 \%$. At last, the proposed RNN-LSTM model has resulted in higher precision, recall, F-measure, and accuracy of $97.04 \%, 97.45 \%, 97.18 \%$, and $97.20 \%$.

Table 6. Result Analysis of Existing with Proposed RNN-LSTM Method.

\begin{tabular}{ccccc}
\hline Methods & Precision & Recall & F-Measure & Accuracy \\
\hline RNN-LSTM & 97.04 & 97.45 & 97.18 & 97.20 \\
\hline Deep Learning & 93.54 & 94.87 & 94.12 & 92.77 \\
\hline DPC-DBN & 95.12 & 94.99 & 95.08 & 94.98 \\
\hline AK-NN & 92.19 & 93.76 & 92.92 & 91.99 \\
\hline Decision Tree & 96.59 & 92.84 & 95.42 & 93.65
\end{tabular}




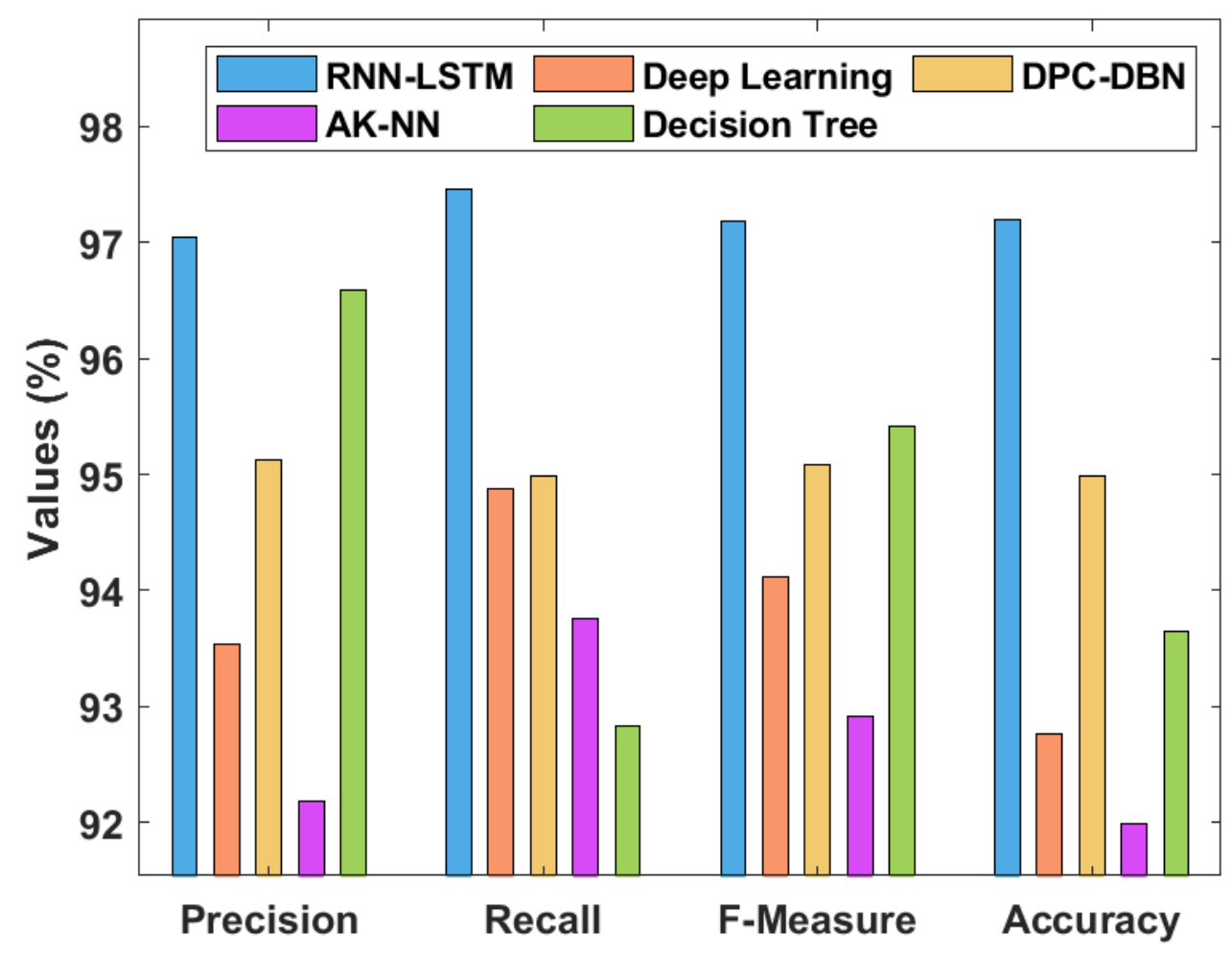

Figure 10. The Comparative results analysis of existing with RNN-LSTM model.

\section{Conclusions}

This paper has developed an efficient GEER-DLAD technique for IoT applications. The presented model permits the IoT devices to exploit the energy efficiently in such a way to rise the network span. Primarily, the IoT devices continuously gather the data. Then, they are compressed by the use of ELC technique. Subsequently, the IoT devices executed the routing technique using MSO algorithm and select the optimal route to destination. Upon the election of routes, the compressed data will be forwarded towards the time. At the time of data transmission, the DLAD technique is applied to detect the occurrence of anomalies in IoT communication networks. A comprehensive experimental validation process takes place and the results guaranteed the improvement of the GEER-DLAD model interms of energy efficiency and detection performance. In future, the detection performance of the DLAD can be improved by the use of learning rate scheduling approaches. Besides, the advanced deep learning architectures can be used instead of RNN-LSTM model for anomaly detection. Furthermore, the local optimal and inefficient global exploration ability of the MSO algorithm can be resolved in our future work. At last, the computational complexity analysis of the proposed model can be investigated as a part of future extension.

Author Contributions: Conceptualization, E.L.L. and A.A.J.; data curation, E.L.L.; formal analysis, E.L.L. and A.A.J.; funding acquisition, C.S. and G.P.J.; investigation, A.F.S.D.; methodology, A.F.S.D. and C.S.; project administration, C.S.; resources, G.P.J.; software, A.A.J.; supervision, G.P.J.; validation, C.S.; visualization, G.P.J.; writing-original draft, A.F.S.D.; writing—review and editing, G.P.J. All authors have read and agreed to the published version of the manuscript.

Funding: This work was supported by the Institute of Information \& Communications Technology Planning \& Evaluation (IITP) grant funded by the Korean Government (MSIT) (Research on AI-Based Cryptanalysis and Security Evaluation) under Grant 2020-0-00126.

Institutional Review Board Statement: Not applicable.

Informed Consent Statement: Not applicable.

Data Availability Statement: The data presented in this study are openly available in https://www. unb.ca/cic/datasets/nsl.html (accessed on 1 March 2021) at [doi: 10.23721/100/1478792]. 
Conflicts of Interest: The authors declare no conflict of interest.

Ethical Approval: This article does not contain any studies with human participants or animals performed by any of the authors.

\section{References}

1. Ortiz, A.M.; Hussein, D.; Park, S.; Han, S.N.; Crespi, N. The cluster between internet of things and social networks: Review and research challenges. IEEE Internet Things J. 2014, 1, 206-215. [CrossRef]

2. Du, Y.; Li, F. Intelligent multi-microgrid energy management based on deep neural network and model-free reinforcement learning. IEEE Trans. Smart Grid 2019, 11, 1066-1076. [CrossRef]

3. Kazerani, M.; Tehrani, K. Grid of Hybrid AC/DC Microgrids: A New Paradigm for Smart City of Tomorrow. In Proceedings of the 2020 IEEE 15th International Conference of System of Systems Engineering (SoSE), Budapest, Hungary, 2-4 June 2020; IEEE: New York, NY, USA, 2020; pp. 175-180.

4. Clausen, T.; Yi, J.; Niktash, A.; Igarashi, Y.; Satoh, H.; Herberg, U.; Lavenu, C.; Lys, T.; Dean, J. The Lightweight on-Demand ad hoc Distance-Vector Routing pRotocol-Next Generation (LOADng); Internet-Draft draft-clausen-lln-loadng-15.txt; IETF Secretariat: Fremont, CA, USA, 2016.

5. Zheng, J.; Lu, C.; Hao, C.; Chen, D.; Guo, D. Improving the generalization ability of deep neural networks for cross-domain visual recognition. IEEE Trans. Cogn. Dev. Syst. 2020. [CrossRef]

6. Zhang, H.; Hao, K.; Gao, L.; Wei, B.; Tang, X. Optimizing Deep Neural Networks through Neuroevolution with Stochastic Gradient Descent. arXiv 2020, arXiv:2012.11184.

7. Yi, J.; Clausen, T.; Igarashi, Y. Evaluation of routing protocol for low power and Lossy Networks: LOADng and RPL. In Proceedings of the 2013 IEEE Conference on Wireless Sensor (ICWISE), Kuching, Malaysia, 2-4 December 2013; pp. 19-24.

8. Sobral, J.V.V.; Rodrigues, J.J.P.C.; Saleem, K.; Al-Muhtadi, J. Performance evaluation of LOADng routing protocol in IoT P2P and MP2P applications. In Proceedings of the 2016 International Multidisciplinary Conference on Computer and Energy Science (SpliTech), Split, Croatia, 13-15 July 2016; pp. 1-6.

9. Sobral, J.V.; Rodrigues, J.J.; Kumar, N.; Zhu, C.; Ahmad, R.W. Performance Evaluation of Routing Metrics in the LOADng Routing Protocol. J. Commun. Softw. Syst. 2017, 13, 87-95. [CrossRef]

10. Hossain, A.K.M.M.; Sreenan, C.J.; Alberola, R.D.P. Neighbour-Disjoint Multipath for Low-Power and Lossy Networks. ACM Trans. Sen. Netw. 2016, 12, 1-25. [CrossRef]

11. Araújo, H.d.S.; Filho, R.H.; Rodrigues, J.J.P.C.; Rabelo, R.d.A.L.; Sousa, N.d.C.; Filho, J.C.C.L.S.; Sobral, J.V.V. A Proposal for IoT Dynamic Routes Selection Based on Contextual Information. Sensors 2018, 18, 353. [CrossRef] [PubMed]

12. Machado, K.; Rosário, D.; Cerqueira, E.; Loureiro, A.A.; Neto, A.; de Souza, J.N. A routing protocol based on energy and link quality for internet of things applications. Sensors 2013, 13, 1942-1964. [CrossRef] [PubMed]

13. Aloi, G.; Caliciuri, G.; Fortino, G.; Gravina, R.; Pace, P.; Russo, W.; Savaglio, C. Enabling IoT interoperability through opportunistic smartphone-based mobile gateways. J. Netw. Comput. Appl. 2017, 81, 74-84. [CrossRef]

14. Javaid, U.; Rasheed, T.; Meddour, D.; Ahmed, T. Adaptive Distributed Gateway Discovery in Hybrid Wireless Networks. In Proceedings of the 2008 IEEE Wireless Communications and Networking Conference, Las Vegas, NV, USA, 31 March-3 April 2008; pp. 2735-2740.

15. Tayeh, G.B.; Makhoul, A.; Laiymani, D.; Demerjian, J. A distributed real-time data prediction and adaptive sensing approach for wireless sensor networks. Pervasive Mob. Comput. 2018, 49, 62-75. [CrossRef]

16. Alieksieiev, V. One approach of approximation for incoming data stream in iot based monitoring system. In Proceedings of the 2018 IEEE Second International Conference on Data Stream Mining Processing (DSMP), Lviv, Ukraine, 21-25 August 2018; pp. 94-97.

17. Azar, J.; Makhoul, A.; Darazi, R.; Demerjian, J.; Couturier, R. On the performance of resource-aware compression techniques for vital signs data in wireless body sensor networks. In Proceedings of the 2018 IEEE Middle East and North Africa Communications Conference (MENACOMM), Jounieh, Lebanon, 18-20 April 2018; pp. 1-6.

18. Gaeta, M.; Loia, V.; Tomasiello, S. Multisignal 1-d compression by F-transform for wireless sensor networks applications. Appl. Soft Comput. 2015, 30, 329-340. [CrossRef]

19. Cheng, L.; Guo, S.; Wang, Y.; Yang, Y. Lifting wavelet compression based data aggregation in big data wireless sensor networks. In Proceedings of the 2016 IEEE 22nd International Conference on Parallel and Distributed Systems (ICPADS), Wuhan, China, 13-16 December 2016; pp. 561-568.

20. Bossen, F. CE1: Table-based bit estimation for CABAC. In Document of ITU-T Q.6/SG16 JCTVC-G763; ITU-T: Geneva, Switzerland, 2011.

21. Zhang, Y.; Lu, C. A highly parallel hardware architecture of table-based CABAC bit rate estimator in an HEVC intra encoder. IEEE Trans. Circuits Syst. Video Technol. 2018, 29, 1544-1558. [CrossRef]

22. Di, S.; Cappello, F. Fast error-bounded lossy HPC data compression with SZ. In Proceedings of the 2016 IEEE International Parallel and Distributed Processing Symposium (IPDPS), Chicago, IL, USA, 23-27 May 2016; pp. 730-739.

23. Azar, J.; Makhoul, A.; Barhamgi, M.; Couturier, R. An energy efficient IoT data compression approach for edge machine learning. Future Gener. Comput. Syst. 2019, 96, 168-175. [CrossRef]

24. Shankar, K.; Perumal, E.; Vidhyavathi, R.M. Deep neural network with moth search optimization algorithm based detection and classification of diabetic retinopathy images. SN Appl. Sci. 2020, 2, 1-10. [CrossRef] 
25. Mohanty, S.N.; Lydia, E.L.; Elhoseny, M.; Al Otaibi, M.M.G.; Shankar, K. Deep learning with LSTM based distributed data mining model for energy efficient wireless sensor networks. Phys. Commun. 2020, 40, 101097. [CrossRef]

26. Datasets NSL-KDD. Available online: https:/ / www.unb.ca/cic/datasets/nsl.html (accessed on 20 July 2020).

27. Diro, A.A.; Chilamkurti, N. Distributed attack detection scheme using deep learning approach for internet of things. Future Gener. Comput. Syst. 2018, 82, 761-768. [CrossRef]

28. Yang, Y.; Zheng, K.; Wu, C.; Niu, X.; Yang, Y. Building an effective intrusion detection system using the modified density peak clustering algorithm and deep belief networks. Appl. Sci. 2019, 9, 44. [CrossRef]

29. Djenouri, Y.; Belhadi, A.; Lin, J.C.W.; Cano, A. Adapted k-nearest neighbors for detecting anomalies on spatio-temporal traffic flow. IEEE Access 2019, 7, 10015-10027. [CrossRef] 